\title{
Editorial
}

\section{Colorectal Cancer Prevention Studies: The Importance of Defining Disease Risk}

\author{
Monica M. Bertagnolli, MD
}

Colorectal cancer (CRC) claims 57,000 lives yearly in the United States. ${ }^{1}$ Although a modest decrease of $1.8 \%$ in the incidence rate for this disease occurred from 1985 to 1995 , incidence rates since then have stabilized. Currently, CRC is the third leading cause of cancer death in both men and women. These statistics persist despite the understanding that the identification and removal of precursor adenomas during screening colonoscopy can prevent CRC. In the landmark National Polyp Study, reported in 1993, ${ }^{2}$ patients having colonoscopic screening with polypectomy at intervals of 1 and 3 years after baseline adenoma removal achieved a reduction in CRC incidence of approximately $76 \%$ to $90 \%$, compared with three reference populations that did not receive polypectomies. Numerous subsequent studies have validated this observation. A particularly dramatic example of effective cancer prevention by close surveillance is provided by Jarvinen et al. ${ }^{3}$ These researchers studied asymptomatic members of families with hereditary nonpolyposis colorectal cancer to determine the effect of CRC screening over a 10-year surveillance period. One group of 133 subjects received screening at 3-year intervals, and a second group of 118 subjects was followed without screening. CRC occurred in $4.5 \%$ of the screened population versus $15 \%$ of the control group, providing a cancer incidence reduction of $62 \%$ that likely occurred because of polypectomy. Equally important, tumor stage at cancer diagnosis was much earlier in the screened population, and no deaths caused by CRC occurred in the screened group, versus five deaths in the control group. Although these data make it clear that CRC screening can dramatically decrease CRC incidence, the significant

Received August 14, 2003; accepted August 22, 2003.

From Brigham \& Women's Hospital, Boston, Massachusetts.

Address correspondence to: Monica M. Bertagnolli, MD, Brigham \& Women's Hospital, 75 Francis St., Boston, MA 02115; Fax: 617-5826177; E-mail: mbertagnolli@ partners.org.

Published by Lippincott Williams \& Wilkins @ 2003 The Society of Surgical Oncology, Inc. drawbacks of high cost and lack of patient acceptance mean that only $10 \%$ to $20 \%$ of the target population receives CRC screening. It is clear that, although we must continue to improve access to and utilization of CRC screening, we must also search for less costly and better accepted methods of cancer prevention.

In addition to adenoma removal, a wealth of data from human epidemiology suggests that dietary or pharmacologic substances can reduce adenoma formation and, in doing so, prevent CRC. ${ }^{4}$ Because of the strength of the adenoma-carcinoma link in human observational studies and in polypectomy trials, the reduction of adenoma incidence is used as a surrogate endpoint for CRC in chemoprevention studies. The report in this issue by $\mathrm{Chu}$ et al. ${ }^{5}$ addresses the incidence of synchronous and metachronous adenoma formation in patients with recently diagnosed CRC. In this study, synchronous disease was defined as adenomas discovered at a baseline colonoscopy that took place within 1.5 years after the resection of an early stage CRC. Recurrent adenomas were those occurring at any time following the baseline colonoscopy. These investigators found that $60 \%$ of patients had synchronous adenomas, a figure consistent with other studies. ${ }^{6,7}$ The 3 -year cumulative adenoma recurrence in this cohort was $35 \%$. This figure is similar to that found in the placebo arm of an aspirin chemoprevention study reported earlier this year by Sandler et al. ${ }^{8}$ These patients also had a history of CRC; however, the time from initial cancer diagnosis to baseline colonoscopy was highly variable. Some patients entered the study immediately following cancer diagnosis, and others 5 or more years following successful treatment. Despite these differences in eligibility, the adenoma recurrence rates in the Sandler et al. ${ }^{8}$ study are comparable. By Kaplan-Meier estimation, the proportion of patients diagnosed with adenomas by 3 years after clearing colonoscopy was approximately $32 \%{ }^{8}$

Any cancer prevention treatment, even one as seemingly innocuous as calcium supplementation, comes with 
risks. At the best, treatment brings the inconvenience of having to take medication on a long-term basis. At the worst, patients at risk who receive chemoprevention can develop a false sense of security and fail to have screening. Other chemopreventive agents have different degrees of risk. For example, nonsteroidal antiinflammatory drugs can increase bleeding complications or be poorly tolerated because of gastric upset. Screening colonoscopy and polypectomy carries a small, but overall significant risk of complications and even death. It is clear that effective, safe use of cancer prevention strategies can only be achieved through accurate identification of cancer and treatment-related risks for individual patients.

The approximately 74,000 patients per year in the United States who have successful treatment for CRC are a particularly important population to target for cancer prevention studies. Not only is the incidence of synchronous and recurrent neoplasia in this group high, these patients may benefit more than most from careful surveillance and chemoprevention. A recent report by Green et al. ${ }^{9}$ shows that, for patients with a history of CRC, second primary CRC are diagnosed at a cumulative incidence of $1.5 \%$ at 5 years, a rate six to seven times greater than the estimated rate of CRC achieved for patients with sporadic adenomas who receive surveillance with polypectomy. In this study, approximately one third of those developing a second primary CRC did so within the first year following surgery, perhaps because of undetected and untreated synchronous disease.

Carcinogenesis in the colon and rectum is a continuum, beginning before the development of an adenoma and progressing through successive stages until a lethal cancer develops. Not all adenomas are equal in their propensity to form advanced tumors, and it is highly likely that a proportion of adenomas can also spontaneously regress. To move the field of CRC chemoprevention forward, it is crucial to continue to define risk factors for CRC cancer and predictors of response to cancer treatment or prevention. Through these studies, we will improve our understanding of tumor biology in ways that will allow us to focus intervention on earlier stages of tumorigenesis and on those patients who will benefit most from treatment of premalignant neoplasia.

\section{REFERENCES}

1. American Cancer Society. Cancer facts and figures, 2003. Atlanta, GA: American Cancer Society; 2003.

2. Winawer SJ, Zauber AG, O'Brien MJ, et al. Prevention of colorectal cancer by colonoscopic polypectomy. The National Polyp Study Workgroup. N Engl J Med 1993;329:1977-81.

3. Jarvinen HJ, Mecklin JP, Sistonen P. Screening reduces colorectal cancer rate in families with hereditary nonpolyposis colorectal cancer. Gastroenterology 1995;108:1405-11.

4. Hawk ET, Limburg PJ, Viner JL. Epidemiology and prevention of colorectal cancer. Surg Clin North Am 2002;82:905-41.

5. Chu DZJ, Chansky K, Alberts DS, et al. Adenoma recurrences after resection of colorectal carcinoma: results from the Southwest Oncology Group 9041 Calcium Chemoprevention Pilot Study. Ann Surg Oncol 2003;10:870-5.

6. Slater G, Fleshner P, Aufses AH Jr. Colorectal cancer location and synchronous adenomas. Am J Gastroenterol 1988;83:832-6.

7. Tate JJ, Rawlinson J, Royle GT, Brunton FJ, Taylor I. Pre-operative or postoperative colonic examination for synchronous lesions in colorectal cancer. Br J Surg 1988;75:1016-8.

8. Sandler RS, Halabi S, Baron JA, et al. A randomized trial of aspirin to prevent colorectal adenomas in patients with previous colorectal cancer. $N$ Engl J Med 2003;348:883-90.

9. Green RJ, Metlay JP, Propert K, et al. Surveillance for second primary colorectal cancer after adjuvant chemotherapy: an analysis of Intergroup 0089. Ann Intern Med 2002;136:261-9. 